\title{
Nuclear Magnetic Relaxation of Oligo- and Poly $(\alpha$-methylstyrene)s in Dilute Solution
}

\author{
Masashi OsA, Hiroshi UedA, Takenao YoshIZAKI, ${ }^{\dagger}$ and Hiromi YAMAKAWA \\ Department of Polymer Chemistry, Kyoto University, Katsura, Kyoto 615-8510, Japan
}

(Received July 30, 2004; Accepted September 29, 2004; Published January 15, 2005)

\begin{abstract}
The spin-lattice relaxation time $T_{1}$ and nuclear Overhauser enhancement NOE were determined for three samples of atactic oligo- and poly ( $\alpha$-methylstyrene)s (a-P $\alpha \mathrm{MS}$ ) with weight-average degree of polymerization $x_{\mathrm{w}}=2,3$, and $67.1 \mathrm{in}$ cyclohexane at $30^{\circ} \mathrm{C}$. A comparison is made of the present data for $T_{1}$ and NOE with the helical wormlike (HW) chain theory, and it is shown that the theory may explain well the data for large $x_{\mathrm{w}}(\gtrsim 10)$. For smaller $x_{\mathrm{w}}$, the rigid sphere model having the radius equal to the apparent root-mean-square radius of gyration of the HW chain fails to give a quantitative explanation of $T_{1}$ in contrast to the case of the relaxation time $\tau_{\Gamma}$ determined from dynamic depolarized light scattering measurements, indicating that the nuclear magnetic relaxation and dynamic depolarized light scattering cannot be described in terms of a common single relaxation time. However, it is shown that there is an effective (mean) magnetic relaxation time $\tau_{\mathrm{M}}$ approximately equal to $0.6 \tau_{\Gamma}$. From a comparison of the present results for $T_{1}$ for a-P $\alpha \mathrm{MS}$ with previous ones for typical flexible polymers, it is also shown that the behavior of $T_{1}$ for them, including a-P $\alpha \mathrm{MS}$, may be consistently explained by the use of $\tau_{\mathrm{M}}$ (proportional to $\tau_{\Gamma}$ ).

[DOI 10.1295/polymj.37.14]

KEY WORDS Poly $(\alpha$-methylstyrene) / Nuclear Magnetic Relaxation / Spin-Lattice Relaxation Time / Nuclear Overhauser Enhancement / Helical Wormlike Chain /
\end{abstract}

In the preceding paper, ${ }^{1}$ we have examined the behavior of the relaxation time $\tau_{\Gamma}$ defined by the reciprocal of the half-width at half-maximum of the spectrum $J_{\Gamma}$ of the excess depolarized component of the light scattered by atactic oligo- and $\operatorname{poly}(\alpha$-methylstyrene)s (a-P $\alpha \mathrm{MS})$ in cyclohexane at $30.5^{\circ} \mathrm{C}(\Theta)$. The quantity $\tau_{\Gamma}$ represents a mean of relaxation (correlation) times of time-correlation functions of the spherical tensor components of the polarizability tensor affixed to the repeat unit of a polymer chain. In the framework of the dynamic theory for the (unperturbed) helical wormlike (HW) chain, ${ }^{2,3}$ those timecorrelation functions are associated with the subspace $L(n)=2(1)$ (of full Hilbert space) spanned by the basis set with the "total angular momentum quantum number" $L=2$ and the number of "excited" subbodies $n=1$. The spin-lattice relaxation time $T_{1}$ and nuclear Overhauser enhancement NOE determined from nuclear magnetic relaxation measurements may also be written in terms of the time-correlation functions of the same class $2(1),{ }^{2,4,5}$ and therefore have a close relation to $\tau_{\Gamma}$. In the present paper as a continuation of the preceding one, ${ }^{1}$ we report results of a nuclear magnetic relaxation study of a-P $\alpha$ MS.

We have already investigated $T_{1}$ and NOE for atactic polystyrene (a-PS), ${ }^{6}$ atactic poly(methyl methacrylate) (a-PMMA), ${ }^{7}$ and isotactic (i-) PMMA, ${ }^{8}$ and compared the results with those for $\tau_{\Gamma},{ }^{6-8}$ and found that $T_{1}$ and NOE are closely correlated with $\tau_{\Gamma}$, as mentioned in the INTRODUCTION in the preceding paper. ${ }^{1}$ Thus the purpose of the present paper is to examine whether such a close correlation holds also for a-P $\alpha$ MS.

\section{EXPERIMENTAL}

\section{Materials}

All the three a-P $\alpha$ MS samples OAMS2, OAMS3, and OAMS67 used in this work are the same as those used in the preceding study of $J_{\Gamma}{ }^{1}$ In Table I are reproduced the values of the weight-average molecular weight $M_{\mathrm{w}}$, weight-average degree of polymerization $x_{\mathrm{w}}$, ratio of $M_{\mathrm{w}}$ to the number-average molecular weight $M_{\mathrm{n}}$, and fraction of racemic diads $f_{\mathrm{r}}$ for the three samples from Table I of ref 1 . The details of their preparation have been described in the EXPERIMENTAL section of the preceding paper. ${ }^{1}$

The solvent cyclohexane used for nuclear magnetic

Table I. Values of $M_{\mathrm{w}}, x_{\mathrm{w}}, M_{\mathrm{w}} / M_{\mathrm{n}}$, and $f_{\mathrm{r}}$ for atactic oligo- and poly( $\alpha$-methylstyrene)s

\begin{tabular}{ccccc}
\hline Sample & $M_{\mathrm{w}}$ & $x_{\mathrm{w}}$ & $M_{\mathrm{w}} / M_{\mathrm{n}}$ & $f_{\mathrm{r}}$ \\
\hline OAMS2 & $2.94 \times 10^{2}$ & 2 & 1 & 0.56 \\
OAMS3 & $4.12 \times 10^{2}$ & 3 & 1 & 0.73 \\
OAMS67 & $7.97 \times 10^{3}$ & 67.1 & 1.04 & 0.72 \\
\hline
\end{tabular}

${ }^{\dagger}$ To whom correspondence should be addressed (E-mail: yoshizaki@molsci.polym.kyoto-u.ac.jp). 
Table II. Values of $T_{1}$ and NOE for OAMS2 in cyclohexane at $30^{\circ} \mathrm{C}$

\begin{tabular}{|c|c|c|}
\hline \multirow{2}{*}{$\begin{array}{l}\text { Carbon } \\
\text { atom no. }\end{array}$} & $\left(T_{1, m m}, T_{1, r m}, T_{1, m r}, T_{1, r r}\right)$ & $\left(\mathrm{NOE}_{m m}, \mathrm{NOE}_{r m}, \mathrm{NOE}_{m r}, \mathrm{NOE}_{r r}\right)$ \\
\hline & $c=0.183 \mathrm{~g} / \mathrm{cm}^{3}$ & $c=0.092 \mathrm{~g} / \mathrm{cm}^{3}$ \\
\hline 5 & $\left(0.90_{9}, 0.89_{3}, 1.0_{1}, 0.98_{6}\right)$ & $\left(3.0_{4}, 2.8_{0}, 2.7_{7}, 2.9_{0}\right)$ \\
\hline 8 & $\left(0.93_{8}, 0.89_{2}, 0.99_{6}, 0.99_{8}\right)$ & $\left(3.0_{6}, 3.0_{4}, 3.1_{0}, 3.0_{3}\right)$ \\
\hline
\end{tabular}

relaxation measurements was purified according to a standard procedure prior to use.

\section{Nuclear Magnetic Relaxation}

$T_{1}$ for ${ }^{13} \mathrm{C}$ was determined for aliphatic carbon atoms of the samples OAMS2, OAMS3, and OAMS67 by the inversion-recovery method with a pulse sequence $\pi-\tau-\pi / 2$ on a JEOL JNM-A500 spectrometer at $125.8 \mathrm{MHz}$. NOE for each of those carbon atoms for the three samples was evaluated from the ratio of the integrated intensity of its peak obtained with complete noise decoupling of protons to that obtained with gated decoupling only during data acquisition. A pulse delay was taken to be longer than 5 times as long as the largest $T_{1}$ of the aliphatic carbon atoms under observation. The pulse delay adopted here is somewhat shorter than the one proposed by Opella et $a l .{ }^{9}$ which is longer than 8 times as long as the largest $T_{1}$. As they have noted, an insufficient pulse delay may lead to an underestimate of NOE for small molecules. As shown in the next section, however, the values of NOE for the sample OAMS2 with the smallest $x_{\mathrm{w}}$ are found to be $c a$. 3 , indicating that the pulse delay adopted here has not led to any appreciable underestimate of NOE.

Measurements were carried out on solutions in cyclohexane at $30^{\circ} \mathrm{C}$ with a lock signal obtained from an external $\mathrm{C}_{6} \mathrm{D}_{12}$ tube. The solutions were not degassed since $T_{1}$ 's of interest rarely exceeded $2 \mathrm{~s}$, as shown later.

\section{RESULTS}

In the case of a-PS 6 (without $\alpha$-methyl group), the nuclear magnetic spins of all main-chain (aliphatic) ${ }^{13} \mathrm{C}$ atoms relax due to the dipolar interactions with bonded hydrogen atoms. In the case of a-P $\alpha \mathrm{MS}$ as well as of a- and i-PMMAs, ${ }^{7,8}$ however, the spins of the methylene ${ }^{13} \mathrm{C}$ atoms relax by the same mechanism as above, while those of the $\alpha-{ }^{13} \mathrm{C}$ atoms do not since the latter have no bonded hydrogen atoms, so that we consider $T_{1}$ and NOE only for the methylene ${ }^{13} \mathrm{C}$ atoms. We note that the nuclear magnetic re- laxation of its methyl ${ }^{13} \mathrm{C}$ atoms does not directly reflect local motions of its main chain, and is not considered, for simplicity.

The values of $T_{1}$ and NOE for the sample OAMS2 in cyclohexane at $30^{\circ} \mathrm{C}$ are given in Table II. The aliphatic carbon atoms have been numbered as explicitly shown in its caption, and the $x$ th carbon atom is designated by $\mathrm{C} x(x=1, \ldots, 10)$. The sample OAMS2 has the two methylene carbon atoms C5 and C8 and is a mixture of the four stereoisomers denoted by $m m, m r, r m$, and $r r$, where $m$ and $r$ denote meso and racemic diads, respectively, and the first and second letters ( $r$ or $m$ ) indicate the stereoisomeric states of the C3-C5-C6 and C6-C8-C9 diads, respectively. In its methylene ${ }^{13} \mathrm{C} \mathrm{NMR}$ spectra, therefore, we are concerned with eight resonance peaks. We have identified each peak by the use of the assignments previously determined for the same sample. ${ }^{10}$ We note that although we could not precisely estimate experimental errors in the raw data, those in the evaluation of $T_{1}$ from the spectra are at most $c a$. $\pm 2 \%$. It is seen that the values of NOE obtained for the sample OAMS2 are $c a .3$ for all ${ }^{13} \mathrm{C}$ atoms, corresponding to the narrowing limit.

In Table III are given the values of $T_{1}$ and NOE for the sample OAMS3 along with its chemical structure with the numbering system for the aliphatic carbon atoms. It has the three methylene carbon atoms $\mathrm{C} 5$, $\mathrm{C}^{\prime}$, and $\mathrm{C} 8$ and is a mixture of the eight stereoisom-

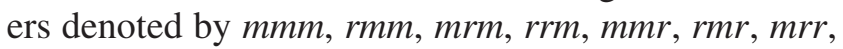
and $r r r$, where the first, second, and third letters indicate the stereoisomeric states of the C3-C5-C6, C6$\mathrm{C} 8{ }^{\prime}-\mathrm{C}^{\prime}$, and $\mathrm{C} 6^{\prime}-\mathrm{C} 8-\mathrm{C} 9$ diads, respectively. In its methylene ${ }^{13} \mathrm{C}$ NMR spectra, therefore, we are in principle concerned with 24 resonance peaks. We have identified each peak by the use of the assignments previously determined for the same sample. ${ }^{10}$ However, the intensities of the 12 peaks for the stereoisomers $\mathrm{mmm}, \mathrm{rmm}, \mathrm{mmr}$, and $\mathrm{rmr}$ are very weak, and therefore we have only considered the peaks for $\mathrm{mrm}$, rrm, mrr, and rrr. We note that the assignments for $\mathrm{mrm}$ and $\mathrm{rrm}$ and also those for $\mathrm{mrr}$ and $\mathrm{rrr}$ can be interchanged. ${ }^{10}$ It is seen that the values of NOE ob- 
Table III. Values of $T_{1}$ and NOE for OAMS3 in cyclohexane at $30^{\circ} \mathrm{C}$

\begin{tabular}{|c|c|c|c|}
\hline \multirow{2}{*}{$\begin{array}{l}\text { Carbon } \\
\text { atom no. }\end{array}$} & \multicolumn{2}{|c|}{$\left(T_{1, m r m}, T_{1, r r m}, T_{1, m r r}, T_{1, r r r}\right)$} & \multirow{2}{*}{$\begin{array}{c}\left(\mathrm{NOE}_{m r m}, \mathrm{NOE}_{r r m}, \mathrm{NOE}_{m r r}, \mathrm{NOE}_{r r r}\right) \\
c=0.092 \mathrm{~g} / \mathrm{cm}^{3}\end{array}$} \\
\hline & $c=0.092 \mathrm{~g} / \mathrm{cm}^{3}$ & $c=0.180 \mathrm{~g} / \mathrm{cm}^{3}$ & \\
\hline 5 & $\left(0.50_{3}, 0.49_{1}, 0.49_{1}, 0.49_{1}\right)$ & $\left(0.42_{8}, 0.41_{8}, 0.42_{3}, 0.41_{8}\right)$ & $\left(2.7_{4}, 2.7_{4}, 2.7_{4}, 2.7_{4}\right)$ \\
\hline $8^{\prime}$ & $\left(0.47_{3}, 0.45_{0}, 0.46_{6}, 0.48_{4}\right)$ & $\left(0.38_{0}, 0.38_{3}, 0.39_{6}, 0.38_{8}\right)$ & $\left(2.7_{6}, 2.8_{0}, 2.8_{9}, 2.8_{4}\right)$ \\
\hline 8 & $\left(0.48_{1}, 0.45_{2}, 0.50_{3}, 0.46_{8}\right)$ & $\left(0.38_{8}, 0.39_{5}, 0.40_{3}, 0.40_{8}\right)$ & $\left(2.9_{4}, 2.7_{8}, 2.8_{7}, 2.8_{6}\right)$ \\
\hline
\end{tabular}

Values of $T_{1}$ and NOE for the stereoisomers $\mathrm{mrm}$ and $\mathrm{rrm}$ and also $\mathrm{mrr}$ and $\mathrm{rrr}$ can be interchanged.

Table IV. Values of $T_{1}$ and NOE for OAMS67 in cyclohexane at $30^{\circ} \mathrm{C}$

\begin{tabular}{ccc}
\hline $\begin{array}{c}c \\
\left(\mathrm{~g} / \mathrm{cm}^{3}\right)\end{array}$ & $\begin{array}{c}T_{1} \\
(\mathrm{~s})\end{array}$ & NOE \\
\hline 0.081 & $0.15_{8}$ & \\
0.108 & $0.16_{4}$ & \\
0.106 & & $1.4_{7}$ \\
\hline
\end{tabular}

tained for the sample OAMS3 are somewhat smaller than those for the sample OAMS2.

For the sample OAMS67, we have identified the peaks of the methylene ${ }^{13} \mathrm{C}$ NMR spectra only for the six kinds of tetrads $\mathrm{mmm}, \mathrm{mmr}, \mathrm{rmr}, \mathrm{mrm}, \mathrm{mrr}$, and $r r r$ by the use of the assignments determined by Inoue et al. ${ }^{11}$ The mean values of $T_{1}$ and NOE are given in Table IV.

Now it is seen from Tables II and III that for the samples OAMS2 and OAMS3, $T_{1}$ and NOE for a given ${ }^{13} \mathrm{C}$ atom depend somewhat on the stereoregularity, as is natural. However, since we are interested in their dependences on $x_{\mathrm{w}}$, we ignore the minor differences between their values for the stereoisomers and simply consider only their mean values for each methylene ${ }^{13} \mathrm{C}$ atom over them. For OAMS2, we consider the averages of mean values so evaluated for $\mathrm{C} 5$ and C8. As for OAMS3, we consider the mean values for $\mathrm{C}^{\prime}$, since we are interested in the motion of the central (or intermediate) part of the a-P $\alpha$ MS chain. We note that for the sample OAMS67 the results obtained are just those for the intermediate methylene ${ }^{13} \mathrm{C}$ atoms. Figure 1 shows plots of the reciprocal of the mean (or averaged) $T_{1}$ against $c$ for the center methylene ${ }^{13} \mathrm{C}$ atoms for the samples OAMS2, OAMS3, and OAMS67. Extrapolation is made to infinite dilution from a pair of data at two concentrations for each sample, following the straight line indicated. As for NOE, we regard the mean (or averaged) values obtained above as those at infinite dilution, for convenience.

The values of $T_{1}$ and NOE thus obtained at infinite

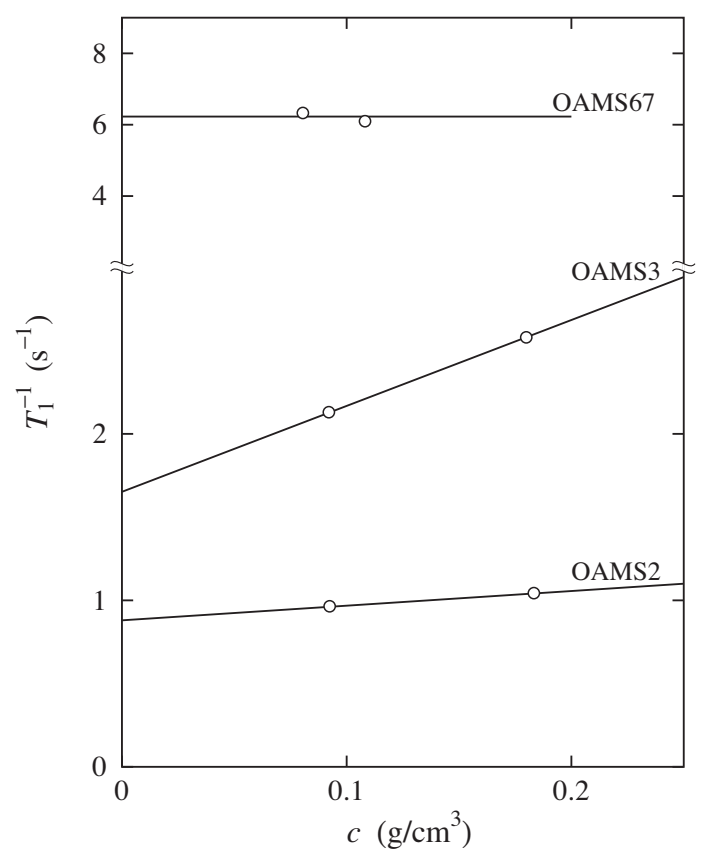

Figure 1. Plots of the reciprocal of the mean $T_{1}$ against $c$ for the center methylene ${ }^{13} \mathrm{C}$ atoms for the three a-P $\alpha$ MS samples in cyclohexane at $30^{\circ} \mathrm{C}$.

Table V. Values of $T_{1}$ and NOE for atactic oligo- and poly ( $\alpha$-methylstyrene)s in cyclohexane at $30^{\circ} \mathrm{C}$ at infinite dilution

\begin{tabular}{lll}
\hline Sample & $\begin{array}{l}T_{1} \\
(\mathrm{~s})\end{array}$ & NOE \\
\hline OAMS2 & $1.1_{4}$ & $2.9_{6}$ \\
OAMS3 & $0.60_{5}$ & $2.8_{4}$ \\
OAMS67 & $0.16_{1}$ & $1.4_{7}$ \\
\hline
\end{tabular}

dilution are summarized in Table V. Note that for flexible polymer chains $T_{1}$ and NOE in general increase with decreasing relaxation time of the orientation of the $\mathrm{C}-\mathrm{H}$ internuclear vector. It is seen that $T_{1}$ and NOE decrease with increasing $x_{\mathrm{w}}$. Considering the previous results for a-PS ${ }^{6}$ and a- and i-PMMAs, ${ }^{7,8}$ the values $0.16_{1} \mathrm{~s}$ and $1.4_{7}$ for OAMS67 may be re- 
garded as the asymptotic values of $T_{1}$ and NOE, respectively, in the limit of $x_{\mathrm{w}} \rightarrow \infty$. We note that although Inoue et al. ${ }^{12}$ reported the values of $T_{1}$ and NOE for a syndiotactic P $\alpha$ MS sample with the viscosity-average molecular weight $1.14 \times 10^{6}$, we have not compared those with the present values, since the solvent condition and the Larmor frequency (strength of external magnetic field) of an NMR spectrometer they adopted are different from ours.

\section{DISCUSSION}

\section{HW Theory}

Before proceeding to make a comparison of the present results of nuclear magnetic relaxation measurements with the HW theory, ${ }^{2,4,5}$ we briefly summarize its relevant results.

We assume that the nuclear magnetic spin (of a methylene ${ }^{13} \mathrm{C}$ atom in the present case) relaxes due to the heteronuclear dipolar interaction between two unlike spins $I$ and $S$, with spin $I$ observed and spin $S$ irradiated (i.e., spins of ${ }^{13} \mathrm{C}$ and ${ }^{1} \mathrm{H}$ atoms, respectively, in the present case, so that $I=S=1 / 2$ ) and with the internuclear distance $r$ between them. Then $T_{1}$ and NOE for the single (discrete) HW chain composed of $N$ identical rigid subbodies may be given $\mathrm{by}^{2,4}$

$$
\begin{aligned}
T_{1}^{-1}= & (1 / 20) K^{2} r^{-6} \\
& \times\left[J_{0}\left(\omega_{S}-\omega_{I}\right)+3 J_{1}\left(\omega_{I}\right)+6 J_{2}\left(\omega_{S}+\omega_{I}\right)\right] \\
\mathrm{NOE}= & 1+\frac{\gamma_{S}}{\gamma_{I}} \\
& \times\left[\frac{6 J_{2}\left(\omega_{S}+\omega_{I}\right)-J_{0}\left(\omega_{S}-\omega_{I}\right)}{J_{0}\left(\omega_{S}-\omega_{I}\right)+3 J_{1}\left(\omega_{I}\right)+6 J_{2}\left(\omega_{S}+\omega_{I}\right)}\right]
\end{aligned}
$$

with

$$
K=\hbar \gamma_{I} \gamma_{S}
$$

where $\gamma_{I}$ and $\gamma_{S}$ are the gyromagnetic ratios of spins $I$ and $S$, respectively, $\omega_{I}$ and $\omega_{S}$ are the respective Larmor angular frequencies, $\hbar$ is Dirac's constant (Planck's constant divided by $2 \pi)$, and $J_{m}(\omega)(m=$ $0,1,2)$ are the spectral density written in the form ${ }^{2,4}$

$$
J_{m}(\omega)=2 \sum_{k=1}^{N}\left(Q_{p k}^{0}\right)^{2} \sum_{j=-2}^{2} \frac{A_{2, k}^{j} \tau_{2, k}^{j}}{1+\left(\omega \tau_{2, k}^{j}\right)^{2}}
$$

with $p$ indicating the subbody number and

$$
\begin{gathered}
Q_{p k}^{0}=[2 /(N+1)]^{1 / 2} \sin [\pi p k /(N+1)] \\
\tau_{2, k}^{j}=1 / \lambda_{2, k}^{j}
\end{gathered}
$$

We note that $J_{m}$ is independent of $m$. The quantities $\lambda_{2, k}^{j}$ in eq 6 are the eigenvalues of the matrix representation of the diffusion operator associated with the subspace 2(1) (of full Hilbert space) spanned by the basis set with the "total angular momentum quantum number" $L=2$ and the number of "excited" subbodies $n=1$, which have already appeared in $J_{\Gamma}$ given by eq 1 (with eq 2 ) of the preceding paper, ${ }^{1}$ and the coefficients $A_{2, k}^{j}$ in eq 4 are given by eq 26 of ref 4 . As in the preceding paper, ${ }^{1}$ we use the augmented eigenvalues $\lambda_{2, k}^{j}$ given by eq 25 of ref 5 which takes partly into account the interactions with the complementary subspace. Thus $\lambda_{2, k}^{j}$ may readily be calculated for given values of $N$ and six HW model parameters. They are the constant differential-geometrical curvature $\kappa_{0}$ and torsion $\tau_{0}$ of the characteristic helix, the static stiffness parameter $\lambda^{-1}$, the bond length $a$, and the translational and rotatory friction coefficients $\zeta_{\mathrm{t}}$ and $\zeta_{\mathrm{r}}$ of the subbody. We note that the coefficient $A_{2, k}^{j}$ depends on the parameters $\kappa_{0}, \tau_{0}$, and $\lambda^{-1}$ and also on the polar and azimuthal angles $\alpha$ and $\beta$ which specify the direction of the $\mathrm{C}-\mathrm{H}$ internuclear vector in the localized coordinate system affixed to the subbody.

\section{Dependences of $T_{1}$ and NOE on $x_{w}$}

Now we proceed to make an analysis of the present results for $T_{1}$ and NOE. Figure 2 shows plots of $n_{\mathrm{CH}} T_{1}$ and NOE against the logarithm of $x_{\mathrm{w}}$ for the center methylene ${ }^{13} \mathrm{C}$ atoms for a-P $\alpha \mathrm{MS}$ in cyclohexane at $30^{\circ} \mathrm{C}$, where $n_{\mathrm{CH}}$ is the number of $\mathrm{C}-\mathrm{H}$ bonds attached to the ${ }^{13} \mathrm{C}$ atom under observation and is equal to 2 in the present case. The unfilled and filled circles represent the observed values of $T_{1}$ and NOE, respectively, given in Table $\mathrm{V}$, and the solid curves represent

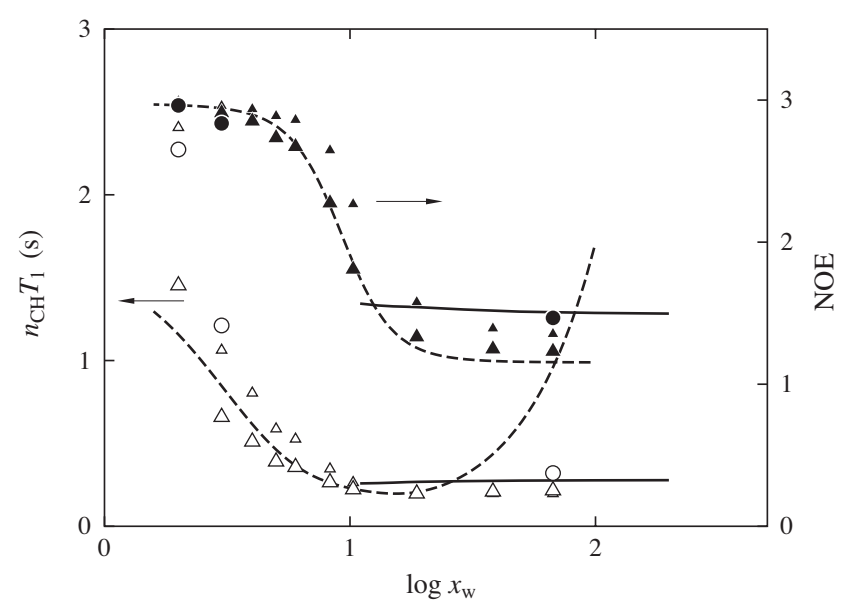

Figure 2. Plots of $n_{\mathrm{CH}} T_{1}$ and NOE against the logarithm of $x_{\mathrm{w}}$ for the center methylene ${ }^{13} \mathrm{C}$ atoms (with $n_{\mathrm{CH}}=2$ ) for a-P $\alpha \mathrm{MS}$ in cyclohexane at $30^{\circ} \mathrm{C}$ : (○) $n_{\mathrm{CH}} T_{1} ;(\bullet)$ NOE. The solid and dashed curves represent the theoretical values for the HW model and the rigid sphere model, respectively. The unfilled and filled large triangles represent the values of $n_{\mathrm{CH}} T_{1}$ and NOE, respectively, calculated with $\tau_{\mathrm{M}}=C \tau_{\Gamma}^{*}$ along with $C=1$, and the small ones represent those with $C=0.6$ (see the text). 
the respective HW theoretical values calculated from eqs 1-6 with the same values of the static and dynamic model parameters as those used in the preceding paper, ${ }^{1}$ i.e., $\lambda^{-1} \kappa_{0}=3.0, \lambda^{-1} \tau_{0}=0.9, \lambda^{-1}=46.8 \AA$, and $\lambda a=0.063(a=2.95 \AA)$ for the static ones and $r_{1} \equiv \zeta_{\mathrm{t}} / 3 \pi \eta_{0} a=1.0$ and $r_{2} \equiv \zeta_{\mathrm{r}} / a^{2} \zeta_{\mathrm{t}}=8.0$ for the dynamic ones, where $\eta_{0}$ is the solvent viscosity. Further, we have adopted the values $90^{\circ}$ and $\pm 55^{\circ}$ for the angles $\alpha$ and $\beta$, respectively, as before. ${ }^{4}$ We note that the coefficients $A_{2, k}^{j}$ in eq 4 and therefore $J_{m}$ given by eq 4 do not depend on the sign of $\beta$ when $\alpha=90^{\circ}$. This means that the two protons of the two bonded hydrogen atoms make equivalent contributions to $T_{1}$, and then $T_{1}$ calculated from eqs $1-6$ with $\beta=55^{\circ}$ is just twice the observed $T_{1}$ and therefore corresponds to the observed $n_{\mathrm{CH}} T_{1}$. For the Larmor angular frequencies $\omega_{I}$ and $\omega_{S}$, we have used the values $2 \pi \times 125.8 \times 10^{6}$ and $2 \pi \times 500.2 \times 10^{6} \mathrm{rad} / \mathrm{s}$, respectively, corresponding to the present measurements. We have also adopted the values $1.09 \AA$ of $r$ and $0.826 \mathrm{cP}$ of $\eta_{0}$ for cyclohexane at $30^{\circ} \mathrm{C}$. The calculation of the theoretical values has been limited to the range of $N\left(=x_{\mathrm{w}}\right) \gtrsim 10$ as in the preceding study of $J_{\Gamma} \cdot{ }^{1}$ For such large $N$, the values of $T_{1}$ and NOE observed for the center (intermediate) methylene carbon atoms are actually the mean values averaged over the position of ${ }^{13} \mathrm{C}$, so that we have presented the theoretical values averaged over $p$, which may be obtained by replacing $\left(Q_{p k}^{0}\right)^{2}$ by $N^{-1}$ in eq 4 .

The HW theoretical values of both $n_{\mathrm{CH}} T_{1}$ and NOE are almost independent of $x_{\mathrm{w}}$ in its range (for $x_{\mathrm{w}} \gtrsim 10$ ) for which the theory is valid. Their asymptotic values in the limit of $x_{\mathrm{w}} \rightarrow \infty$ are $0.278 \mathrm{~s}$ and 1.50 , respectively, which are in rather good agreement with the values $0.32_{2} \mathrm{~s}$ and $1.4_{7}$, respectively, observed for the sample OAMS67.

Next, in order to examine the behavior of $T_{1}$ and NOE for small $x_{\mathrm{w}}$, we consider the rigid sphere mod$\mathrm{el}^{2}$ which has the radius equal to the apparent rootmean-square radius of gyration $\left\langle S^{2}\right\rangle_{\mathrm{s}}^{1 / 2}$ of the HW chain as before ${ }^{1}$ but to which a $\mathrm{C}-\mathrm{H}$ internuclear vector is affixed in the present case. $T_{1}$ and NOE may then be given by eqs 1 and 2, respectively, with $J_{m}$ given by ${ }^{13}$

$$
J_{m}(\omega)=\frac{2 \tau_{\mathrm{M}}}{1+\left(\omega \tau_{\mathrm{M}}\right)^{2}}
$$

where $\tau_{\mathrm{M}}$ is identical to the relaxation time $\tau_{\Gamma}$ associated with $J_{\Gamma}$ given by eq 6 of the preceding paper $^{1}$ and is given by

$$
\tau_{\mathrm{M}}=4 \pi \eta_{0}\left\langle S^{2}\right\rangle_{\mathrm{s}}^{3 / 2} / 3 k_{\mathrm{B}} T
$$

with $k_{\mathrm{B}}$ the Boltzmann constant and $T$ the absolute temperature. Note that $T_{1}$ so obtained corresponds to the observed $n_{\mathrm{CH}} T_{1}$ as in the case of the HW theoret- ical values above. As noted in the preceding paper, ${ }^{1}$ $\left\langle S^{2}\right\rangle_{\mathrm{s}}^{1 / 2}$ is defined as the coefficient of the squared scattering vector $k^{2}$ in the expansion of the scattering function determined from small-angle X-ray scattering measurements and may be regarded as the mean-square radius of gyration of the excess electron density. It may then be given by

$$
\left\langle S^{2}\right\rangle_{\mathrm{s}}=\left\langle S^{2}\right\rangle+S_{\mathrm{c}}^{2}
$$

where $\left\langle S^{2}\right\rangle$ is the (usual) mean-square radius of gyration of the HW chain contour of total length $L$ and is given by eq 5 of ref 14 (or eq 4.83 of ref 2 in units of $\lambda^{-1}$ ) and $S_{\mathrm{c}}$ is the (effective) radius of gyration of the cross section of the excess electron density distributed around the chain contour and has already been evaluated to be $\sqrt{10.7} \AA$ for a-P $\alpha$ MS. ${ }^{14}$

In Figure 2, the values of $n_{\mathrm{CH}} T_{1}$ and NOE calculated from eqs 1 and 2, respectively, with eqs 7-9 and with the above values of $\omega_{I}, \omega_{S}, r$, and $\eta_{0}$ are represented by the respective dashed curves. The differences between the observed and calculated values of $T_{1}$ for the samples OAMS2 and OAMS3 are not very small, especially for the former. This disagreement implies that $T_{1}$ cannot be explained in terms of the single relaxation time $\tau_{\Gamma}$ for a-P $\alpha$ MS as well as for a- and i-PMMAs ${ }^{7,8}$ (with a given $x_{\mathrm{w}}$ ) in contrast to the case of a-PS, ${ }^{6}$ although such an explanation is possible for $J_{\Gamma}$ for all of them.

As before, ${ }^{6-8}$ we then examine values of $T_{1}$ and NOE calculated from eqs 1 and 2, respectively, with eq 7, where we equate $\tau_{\mathrm{M}}$ to the scaled $\tau_{\Gamma}$ in cyclohexane at $30^{\circ} \mathrm{C}$, which we denote by $\tau_{\Gamma}^{*}$, taking account of the differences in $\eta_{0}$ and $T$ by the use of the equation

$$
\tau_{\Gamma}^{*}=\left[\left(\eta_{0} / T\right)_{30} /\left(\eta_{0} / T\right)_{30.5}\right] \tau_{\Gamma}
$$

with the observed values of $\tau_{\Gamma}$ in cyclohexane at $30.5^{\circ} \mathrm{C}$ given in Table II of the preceding paper. ${ }^{1}$ If $T_{1}$ and NOE could be explained in terms of this $\tau_{\Gamma}^{*}$, their calculated values would agree well with the observed ones. The values of $n_{\mathrm{CH}} T_{1}$ and NOE so calculated for all the samples investigated in the preceding paper ${ }^{1}$ are represented by the unfilled and filled large triangles, respectively, in Figure 2. Except for the value $(\simeq 3)$ of NOE in the narrowing limit, the calculated values (large triangles) are appreciably smaller than the observed ones (circles) as in the cases of a- and i-PMMAs ${ }^{7,8}$ and in contrast to the case of a-PS. ${ }^{6}$

As previously mentioned, ${ }^{7}$ the above discrepancy may be regarded as arising from the differences in relative weights of the eigenvalues $\lambda_{2, k}^{j}$ between $J_{\Gamma}$ in the preceding paper $^{1}$ and $J_{m}$ in the present paper and also those among a-P $\alpha$ MS, a-PS, and a- and i-PMMAs. Thus we simply relate an effective mean (single) magnetic relaxation time $\tau_{\mathrm{M}}$ to $\tau_{\Gamma}^{*}$ by 


$$
\tau_{\mathrm{M}}=C \tau_{\Gamma}^{*}
$$

as before, ${ }^{7,8}$ where $C$ is a constant independent of $x_{\mathrm{w}}$ chosen so that the values of $T_{1}$ and NOE calculated from eqs 1 and 2, respectively, with eqs 7, 10, and 11 (and with the observed $\tau_{\Gamma}$ ) are close to the observed ones. In Figure 2, the unfilled and filled small triangles represent the values so calculated with $C=0.6$, which happens to be the same as that previously used for a- and i-PMMAs. ${ }^{7,8}$ As in those cases, these calculated values agree well with the observed ones except for $T_{1}$ at large $x_{\mathrm{w}}$, for which the former values are still $c a .40 \%$ smaller than the latter.

\section{Comparison with Other Flexible Polymers}

Finally, in this subsection we compare the present values of $T_{1}$ for the methylene ${ }^{13} \mathrm{C}$ atoms for a-P $\alpha \mathrm{MS}$ with the previous ones for a-PS ${ }^{6,7}$ and a- and iPMMAs. ${ }^{7,8}$ Unfortunately, however, the present values were determined at $125.8 \mathrm{MHz}$ but the previous ones at $100.4 \mathrm{MHz}$, and therefore the former cannot be directly compared with the latter. Thus we try to estimate the values of $T_{1}$ for ${ }^{13} \mathrm{C}$ for the a-P $\alpha \mathrm{MS}$ at $100.4 \mathrm{MHz}$ by the use of a maneuver on the basis of the theoretical values for the rigid sphere model for the samples OAMS2 and OAMS3 and the HW model for the sample OAMS67. That is, we consider that the value of $T_{1}$ at $100.4 \mathrm{MHz}$ is given by the one at $125.8 \mathrm{MHz}$ multiplied by the ratio of the theoretical value of $T_{1}$ calculated at $100.4 \mathrm{MHz}$ to that at $125.8 \mathrm{MHz}$. The values of the ratio calculated for both samples OAMS2 and OAMS3 are 0.99 and that for the sample OAMS67 is 0.78 , leading to the values $1.1_{3}, 0.59_{9}$, and $0.12_{6} \mathrm{~s}$ of $T_{1}$ at $100.4 \mathrm{MHz}$ for the samples OAMS2, OAMS3, and OAMS67, respectively.

Figure 3 shows double-logarithmic plots of $n_{\mathrm{CH}} T_{1}$ (in s) against $\tau_{\mathrm{M}}$ (in s) for the center methylene ${ }^{13} \mathrm{C}$ atoms (with $n_{\mathrm{CH}}=2$ ) at $100.4 \mathrm{MHz}$ for a-P $\alpha \mathrm{MS}$ in cyclohexane at $30^{\circ} \mathrm{C}$ (unfilled circles), a-PS in cyclohexane at $40^{\circ} \mathrm{C}$ (filled circles), ${ }^{6,7}$ a-PMMA in acetonitrile at $44^{\circ} \mathrm{C}$ (filled triangles), ${ }^{7}$ and i-PMMA in acetonitrile at $35^{\circ} \mathrm{C}$ (filled inverted triangles), ${ }^{8}$ where the values of $\tau_{\mathrm{M}}$ for the respective polymers have been calculated from eq 11 with the values of $\tau_{\Gamma}^{*}$ and with $C=0.6$ for a-P $\alpha$ MS and a- and i-PMMAs ${ }^{7,8}$ and $C=$ 1 for a-PS., 6 The heavy and light dashed curves represent the $\mathrm{HW}$ theoretical values for a-P $\alpha \mathrm{MS}$ and aPS, respectively, and the heavy and light dot-dashed curves represent those for a- and i-PMMAs, respectively, all at $100.4 \mathrm{MHz}$. The right-end point of each curve bounded by the vertical line segment with the shade corresponds to the asymptotic value of $\tau_{\Gamma}$ in the limit of $x_{\mathrm{w}} \rightarrow \infty$, and the other end point corresponds to $x_{\mathrm{w}}=10$. The dotted curve represent the the-

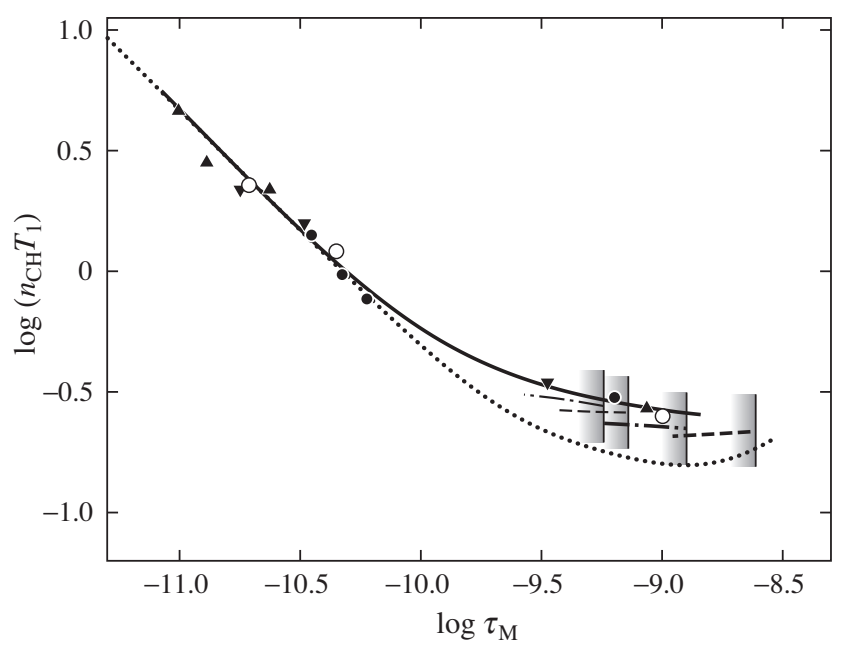

Figure 3. Double-logarithmic plots of $n_{\mathrm{CH}} T_{1}$ (in s) against $\tau_{\mathrm{M}}$ (in s) for the center methylene ${ }^{13} \mathrm{C}$ atoms (with $n_{\mathrm{CH}}=2$ ) at 100.4 MHz: (○) present data for a-P $\alpha \mathrm{MS}$ in cyclohexane at $30^{\circ} \mathrm{C}$; (-) previous data for a-PS in cyclohexane at $40^{\circ} \mathrm{C} ; 6,7$ $(\boldsymbol{\Delta})$ previous data for a-PMMA in acetonitrile at $44{ }^{\circ} \mathrm{C} ;{ }^{7}(\boldsymbol{\nabla})$ previous data for i-PMMA in acetonitrile at $35^{\circ} \mathrm{C} .{ }^{8}$ The heavy and light dashed curves represent the HW theoretical values for a$\mathrm{P} \alpha \mathrm{MS}$ and a-PS, respectively, the heavy and light dot-dashed curves represent those for a- and i-PMMAs, respectively, the dotted curve represents the theoretical values for the rigid sphere model, and the solid curve connects smoothly the data points (see the text).

oretical values calculated from eq 1 with eq 7 for the rigid sphere model at $100.4 \mathrm{MHz}$. It is seen that all the data points for a-P $\alpha \mathrm{MS}$, a-PS, and a- and i-PMMAs form nearly a single-composite curve, as shown by the solid curve, and follow the dotted curve for small $\tau_{\mathrm{M}}$ [for $\log \left(n_{\mathrm{CH}} T_{1}\right) \gtrsim-0.2$ ], as was expected.

\section{CONCLUSIONS}

The spin-lattice relaxation time $T_{1}$ and nuclear Overhauser enhancement NOE were determined for the methylene ${ }^{13} \mathrm{C}$ atoms for a-P $\alpha \mathrm{MS}$ in cyclohexane at $30^{\circ} \mathrm{C}$. It has been shown that the $\mathrm{HW}$ theory may explain well their behavior for large $x_{\mathrm{w}}(\gtrsim 10)$. In contrast to the case of the preceding study of dynamic depolarized light scattering, ${ }^{1}$ the rigid sphere model for smaller $x_{\mathrm{w}}$ fails to give a quantitative explanation of $T_{1}$, indicating that the nuclear magnetic relaxation and dynamic depolarized light scattering cannot be described in terms of a common single relaxation time. However, it has been shown that there is an effective (mean) magnetic relaxation time $\tau_{M}$ approximately equal to $0.6 \tau_{\Gamma}$, and also that the behavior of $T_{1}$ for typical flexible polymers, including a-P $\alpha \mathrm{MS}$, may be consistently explained by the use of $\tau_{M}$ (proportional to $\tau_{\Gamma}$ ). 
Acknowledgment. This research was supported in part by the 21 st century COE program "COE for a United Approach to New Materials Science" from the Ministry of Education, Culture, Sports, Science, and Technology, Japan.

\section{REFERENCES}

1. H. Ueda, M. Osa, T. Yoshizaki, and H. Yamakawa, Polym. J., 37, 7 (2005).

2. H. Yamakawa, "Helical Wormlike Chains in Polymer Solutions,” Springer, Berlin, 1997.

3. T. Yoshizaki and H. Yamakawa, J. Chem. Phys., 99, 9145 (1993).

4. H. Yamakawa and M. Fujii, J. Chem. Phys., 81, 997 (1984).

5. H. Yamakawa, T. Yoshizaki, and M. Fujii, J. Chem. Phys., 84, 4693 (1986).
6. Y. Takaeda, T. Yoshizaki, and H. Yamakawa, Macromolecules, 27, 4248 (1994).

7. Y. Takaeda, T. Yoshizaki, and H. Yamakawa, Macromolecules, 28, 682 (1995).

8. Y. Naito, N. Sawatari, Y. Takaeda, T. Yoshizaki, and H. Yamakawa, Macromolecules, 30, 2751 (1997).

9. S. J. Opella, D. J. Nelson, and O. Jardetzky, J. Chem. Phys., 64, 2533 (1976).

10. M. Osa, M. Sumida, T. Yoshizaki, H. Yamakawa, K. Ute, T. Kitayama, and K. Hatada, Polym. J., 32, 361 (2000).

11. Y. Inoue, A. Nishioka, and R. Chûjô, Makromol. Chem., 156, 207 (1972).

12. Y. Inoue, Y. Kawamura, and T. Konno, Polymer, 23, 817 (1982).

13. I. Solomon, Phys. Rev., 99, 559 (1955).

14. M. Osa, T. Yoshizaki, and H. Yamakawa, Macromolecules, 33, 4828 (2000). 